\title{
Evaluation of changes in choroidal thickness after surgical implantation of collamer lens in patients with different degrees of high myopia
}

\author{
FANGLIN HE* , JIE YANG* ${ }^{*}$ RENBING JIA and JING ZHANG \\ Department of Ophthalmology, Shanghai Key Laboratory of Orbital Disease and Ocular Oncology, \\ Shanghai Ninth People's Hospital, Shanghai Jiaotong University School of Medicine, Shanghai 200011, P.R. China
}

Received February 13, 2019; Accepted July 5, 2019

DOI: $10.3892 /$ etm.2019.7831

\begin{abstract}
The aim of the present study was to investigate the potential changes in the choroidal thickness (CT) after surgical implantation of collamer lens (ICL) and to determine whether the variations in CT were associated with the degree of myopia. In the study, 98 eyes from 98 myopia patients were divided into two groups according to the degree of myopia: High myopia and super-high myopia. All eyes were measured using the swept-source optical coherence tomography (SS-OCT) technique. CT and CT variations were also recorded. The foveal CT increased significantly in high-myopia patients at $2 \mathrm{~h}$ after surgery and 3 months after surgery; the same tendency was observed in the inner nasal CT and outer nasal CT at the same time-points. In patients with super-high myopia, the subfoveal CT increased significantly at $2 \mathrm{~h}$ and 3 months after surgery compared with the pre-operative values. No statistically significant differences were obtained in any of the nine different choroidal regions evaluated at post-operative week 1 and post-operative month one. Furthermore, the increase in the subfoveal CT in the super-myopia group was significantly higher than that in the high-myopia group at $2 \mathrm{~h}$ and at 3 months after ICL. The results of the present study indicated that the CT significantly increased $2 \mathrm{~h}$ after the surgery and then reached a peak at 3 months, particularly in the subfoveal and nasal areas. A higher degree of myopia was associated with greater subfoveal choroidal changes.
\end{abstract}

Correspondence to: Professor Jing Zhang or Professor Renbing Jia, Department of Ophthalmology, Shanghai Key Laboratory of Orbital Disease and Ocular Oncology, Shanghai Ninth People's Hospital, Shanghai Jiaotong University School of Medicine, 639 Zhi Zao Ju Road, Huangpu, Shanghai 200011, P.R. China

E-mail: zhjingty@126.com

E-mail: renbingjia@sjtu.edu.cn

${ }^{*}$ Contributed equally

Key words: choroidal thickness, high myopia, super-high myopia, ICL surgery, degree

\section{Introduction}

The prevention and treatment of myopia have always been a public health concern throughout the world. Recently, the incidence rate of the disease has increased (1), particularly that of high myopia. Use of the implantable collamer lens (ICL), a posterior chamber phakic intraocular lens, has been reported as a safe, effective and reversible approach, which may serve as an appropriate alternative for patients wishing to have refractive surgery, but it is not suitable for corneal refractive surgery due to the high degree of myopia with lower cornea thickness. However, patients must be made aware of possible complications, including increased intraocular pressure (IOP) and inflammation $(2,3)$, which may influence the chorioretinal regions. In previous studies, the safety, efficacy and predictability of these refractive surgeries have been assessed by the change of visual function $(2,4)$ and ocular anterior segment structure $(4,5)$. However, the change of the ocular posterior segment, particularly the choroid, has not been evaluated following ICL surgery. The focus of myopia-associated research has gradually shifted from the dioptric media of the anterior segment to the tissues of the posterior segment, including the choroid.

Unlike patients with emmetropia, those with high myopia have a long axial length that leads to gradual changes in choroidal thickness (CT) and a progressive loss of visual sensitivity. As indicated in previous studies, the choroid expands or thins in myopic and highly myopic eyes (6-8), a change which has a marked positive correlation with the increase of myopia. The choroid is known as the source of numerous vision-threatening diseases (9). A thinner choroid may be instrumental in the onset and progression of severe myopia-associated diseases. During the process of myopic recovery in chicks, the choroid compensates by becoming thicker $(10,11)$. In pediatric patients with myopia wearing overnight orthokeratology contact lens, the thinning CT was recovered due to a reduction or neutralization of the myopiogenic stimulus to eye growth (12). However, a change in the $\mathrm{CT}$ after correcting myopic refractive error by ICL surgery in humans has not been previously reported.

The recently developed swept-source optical coherence tomography (SS-OCT) uses a longer wavelength of $\sim 1,050 \mathrm{~nm}$ as the light source. This novel technique may capture 256 raster scan images covering the entire macula to generate a CT map 
in a single session. In the present study, each choroidal layer was detected automatically using this technique. In addition, the 'follow-up' function ensured that the same choroidal areas were being scanned after surgery, which suggested that the present data are more reliable than those of previous studies. The aim of the present study was to evaluate the potential changes in CT after ICL surgery by using the SS-OCT technique, and to determine whether the variations of the $\mathrm{CT}$ were associated with the degree of myopia.

\section{Materials and methods}

Subjects. The present cross-sectional observational pilot study included 98 eyes from 98 myopia patients recruited between October 2017 and September 2018 at the Department of Ophthalmology of the Ninth People's Hospital (Shanghai Jiao Tong University of Medicine, Shanghai, China). The study was approved by the University Investigational Review Board (Shanghai, China).

The patients had no other ophthalmic or systemic diseases and were eligible for an ICL. The following inclusion criteria were used: i) Diagnosed with high myopia, refractive diopter (D) range from -6.00 to $18.00 \mathrm{D}$; ii) anterior chamber depth $>2.80 \mathrm{~mm}$; central corneal endothelial cell count $>2,000$ cells $/ \mathrm{mm}$, iii) minimum age of 22 years and ability to return for a 3-month follow-up; and iv) the patient volunteered to participate in the study and signed the consent form. Patients aged $>45$ years with unrealistic expectations (13), IOP $>21 \mathrm{~mm} \mathrm{Hg}$ or a history of ocular surgery were excluded, as were cases with uveitis, cataract, diabetes mellitus and other ocular or systemic autoimmune diseases.

Eyes were divided into the following two groups according to the degree of myopia: High myopia, refractive D range from -6.00 to $-10.00 \mathrm{D}$; and super-high myopia, refractive D range from -10.00 to $-18.00 \mathrm{D}$. All subjects underwent SS-OCT (DRI-1; Topcon), as well as comprehensive ophthalmologic examinations prior to surgery and at $2 \mathrm{~h}, 1$ day, 1 week, 1 and 3 months after surgery, including corrected distance visual acuity tests, IOP measurements, axial length (AL) determinations, slit lamp evaluations and fundus examinations. The diurnal variations were considered in the CT. Prior to surgery, the OCT images were obtained at 9 a.m. and the time of operation was at 10 a.m. The first post-operative exam was $2 \mathrm{~h}$ after surgery and the subsequent follow-ups were all at 9:00 a.m. The subjects' characteristics, including age, sex and existence of associated ocular diseases were recorded. All patients were examined by the same physician $(\mathrm{FH})$.

SS-OCT scanning protocol. In the present study, all enrolled eyes were examined by SS-OCT (DRI-1; Topcon) using a light source with a wavelength-tunable laser centered at 1,050 nm with a $1,000-\mathrm{Hz}$ repetition rate. To obtain the data, the $3 \mathrm{D}$ macular volumetric raster scan protocol, which covered a 6x6 mm macular area centered on the fovea, was selected. This protocol may acquire a 3D imaging set consisting of 512 A-scans and 256 B-scans (Fig. 1).

The OCT images captured were assigned to four categories (superior, inferior, nasal and temporal). The CT was measured between the retinal pigment epithelium-Bruch's membrane complex and choroid-scleral interface. An ETDRS-style topographic map of CT was generated automatically by built-in segmentation software. After surgery, OCT images were obtained using the 'follow-up' function to ensure the same choroidal areas were being scanned. The program divides the area into three concentric rings as follows: An inner $1.0-\mathrm{mm}$ ring, an intermediate $3.0-\mathrm{mm}$ ring and an outer $6.0-\mathrm{mm}$ ring. The parafoveal 3.0 - or $6.0-\mathrm{mm}$ ring was also subdivided into four quadrants; the data for each section represented the mean of the respective relative area. To obtain accurate data, each eye was measured twice and the data were averaged for analysis. All of the OCT images were modified regarding their segmentation lines if any automated segmentation errors occurred.

Surgical procedure. Every surgery was performed by the same experienced surgeon (JZ). Mydriasis agents (Tropicamide Eye Drops, Santen) were instilled four times at 10-min intervals prior to surgery. A 3-mm temporal corneal incision was created with a diamond knife. Viscoelastic material was injected in the anterior chamber prior to implanting the IOL. An injector cartridge (STAAR Surgical) was used to insert the ICL V4c with a $0.36-\mathrm{mm}$ central artificial hole (Hole ICL ${ }^{\mathrm{TM}}$ ). The four footplates of the ICL were placed on the ciliary sulcus behind the iris with two manipulators along the $180^{\circ}$ axis. Viscoelastic material was completely removed using buffered saline. The ICL position was verified prior to finalization of the operation. Post-operatively, $0.3 \%$-dexamethasone and $0.1 \%$ tobramycin (Tobradex) eye drops were administered topically three times daily for 1 week.

Statistical analysis. Analysis of variance (ANOVA) and Pearson's $\chi^{2}$ test were used to compare the clinical characteristics between the two groups in different groups. Changes in CT at different time-points in each group were assessed by repeated-measures one-way analysis of variance followed by Tukey's post-hoc test, and independent-samples t-tests (two groups) were applied to analyze significant differences between the two myopia groups. All analyses were performed using the statistical software package GraphPad Prism (version 7.00 for Windows; GraphPad Software Inc.). $\mathrm{P}<0.05$ was considered to indicate a statistically significant difference. Values are expressed as the mean \pm standard deviation.

\section{Results}

Clinical features of patients. In the present study, 42 eyes with high myopia with refractive $\mathrm{D}$ between -6.00 and $-10.00 \mathrm{D}$, and 56 eyes with super-high myopia with refractive D over $-10.00 \mathrm{D}$ were assessed. The high-myopia group consisted of 26 females and 16 males with a mean age of $25.88 \pm 4.04$ years, and the super-high-myopia group consisted of 36 females and 20 males with a mean age of $26.45 \pm 5.02$ years. The mean AL was $25.59 \pm 0.92 \mathrm{~mm}$ in the high-myopia group and $27.74 \pm 1.12 \mathrm{~mm}$ in the super-high myopia group. In the high-myopia group, the mean spherical equivalent (SE) of the refractive error was $-7.91 \pm 2.37 \mathrm{D}$ prior to surgery. At one month after the surgery, the SE was $-0.56 \pm 0.37 \mathrm{D}$ and at 3 months post-operatively, the $\mathrm{SE}$ was $-0.31 \pm 0.32 \mathrm{D}$. In the super-high-myopia group, the mean $\mathrm{SE}$ of the refractive error was $-14.91 \pm 2.17 \mathrm{D}$ prior to surgery. At one month after the surgery, the SE was $-0.62 \pm 0.37 \mathrm{D}$, 

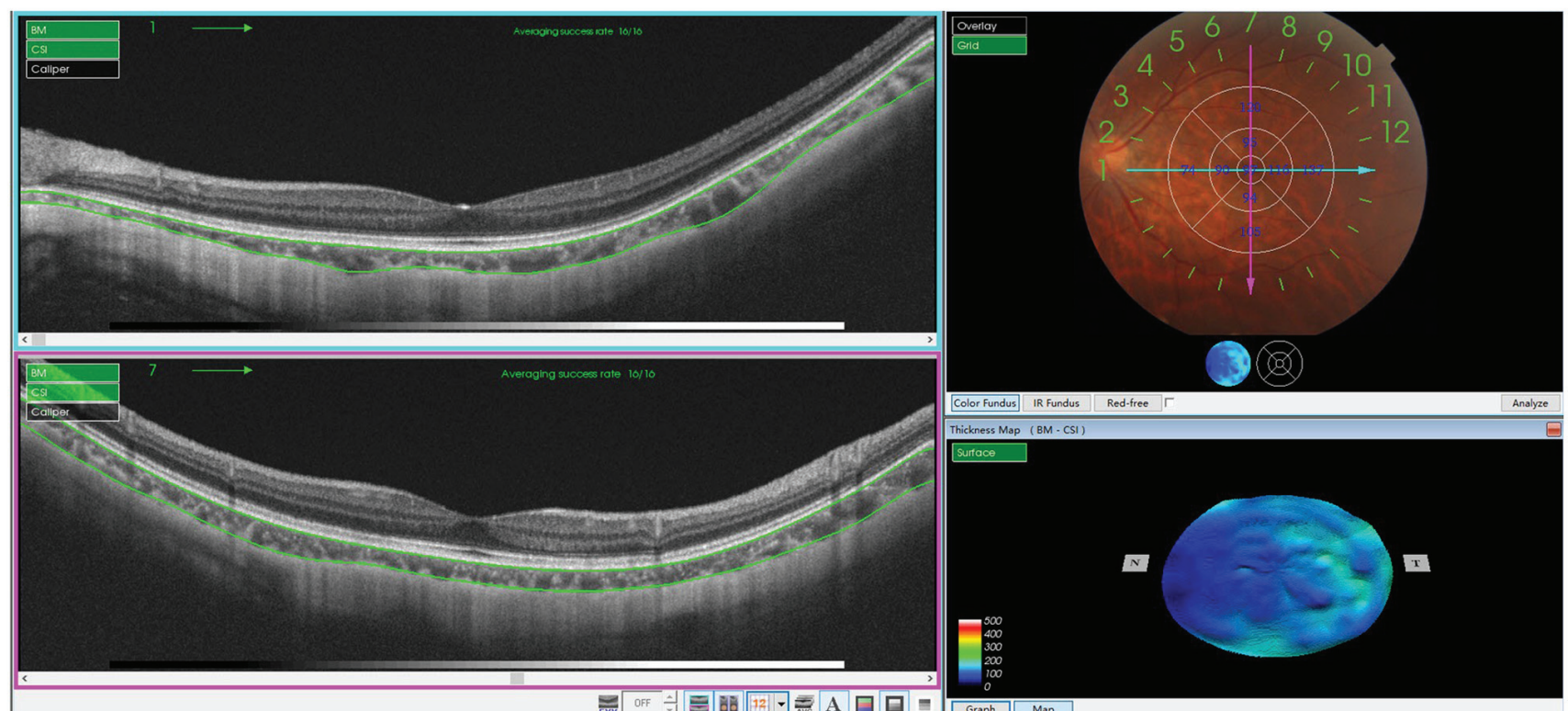

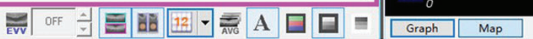

Figure 1. Swept-source optical coherence tomography scanning. Top and bottom left: The choroid was automatically defined as the layer between the retinal pigment epithelium and the chorioscleral interface (green line). Bottom right: CT map of the area corresponding to myopia. Top right: Study layout area and the mean regional CT calculated for the 9 sectors of the layout using the 3D macular volumetric raster scan protocol of swept-source optical coherence tomography. The units for the blue numbers are ' $\mu \mathrm{m}$ '; BM, Bruch's membrane; CSI, Chorioscleral interface; N, Nasal side; T, Temporal side; CT, choroidal thickness.

and at 3 months post-operatively, the SE was $-0.41 \pm 0.32 \mathrm{D}$. The average washing time for each group was $20 \mathrm{sec}$. Table I summarizes the demographic and clinical characteristics of the participants. There were no significant differences in age and sex between the subject groups.

CT changes. The CT changes in the high-myopia group and super-high-myopia group are provided in Tables II and III. Compared with the pre-operative values, the foveal CT was significantly increased in the high-myopia patients at $2 \mathrm{~h}$ after surgery $(203.18 \pm 36.20$ vs. $223.36 \pm 35.12 \mu \mathrm{m}, \mathrm{P}=0.025)$ and at 3 months after surgery $(203.18 \pm 36.20$ vs. $227.43 \pm 35.64 \mu \mathrm{m}$; $\mathrm{P}=0.010)$. The same tendency was observed in the inner nasal CT (2 h, 184.64 \pm 28.30 vs. $198.69 \pm 28.31 \mu \mathrm{m}, \mathrm{P}=0.041$; 3 months, $184.64 \pm 28.30$ vs. $202.74 \pm 31.36 \mu \mathrm{m}, \mathrm{P}=0.016)$ and outer nasal CT (2 h, 167.07 \pm 32.04 vs. $183.44 \pm 30.69 \mu \mathrm{m}$, $\mathrm{P}=0.047 ; 3$ months, $167.07 \pm 32.04$ vs. $185.72 \pm 30.04, \mathrm{P}=0.016$ ) at the same time-points. Fig. 2 presents the CT of the fovea and nasal side at different time-points in the high-myopia group.

In patients with super-high myopia, the CT increased significantly at $2 \mathrm{~h}$ and 3 months after surgery compared with the pre-operative values $(2 \mathrm{~h}, 180.24 \pm 42.89$ vs. $211.48 \pm 43.13 \mu \mathrm{m}$, $\mathrm{P}=0.007 ; 3$ months, $180.24 \pm 42.89$ vs. $219.38 \pm 44.53 \mu \mathrm{m}$, $\mathrm{P}=0.002$ ), and the same tendency was observed in the inner nasal CT $(2 \mathrm{~h}, 169.79 \pm 41.21$ vs. $187.60 \pm 39.56 \mu \mathrm{m}, \mathrm{P}=0.047$; 3 months, $169.79 \pm 41.21$ vs. $189.78 \pm 40.21 \mu \mathrm{m}, \mathrm{P}=0.041)$ and outer nasal CT $(2 \mathrm{~h}, 151.20 \pm 35.13$ vs. $168.23 \pm 34.75 \mu \mathrm{m}$, $\mathrm{P}=0.044 ; 3$ months, $151.20 \pm 35.13$ vs. $170.22 \pm 34.54, \mathrm{P}=0.043$ ) at the same time-points. Fig. 3 presents the CT of the fovea and nasal side at different time-points in the super-high myopia group.

The mean CTs of all areas at the pre-operative time-point and at 1 week post-operatively were similar within each group for both groups. At 1 month post-operatively, the mean CTs in the nine regions were slightly thicker than the corresponding pre-operative values, but no significant difference was observed between the two groups.

Differences in CT variation between the two groups. The CT variation in the two groups is presented in Table IV. The post-operative increase in the subfoveal $\mathrm{CT}$ from baseline in the super-high myopia group was significantly higher than that in the high-myopia group at $2 \mathrm{~h}$ after ICL surgery $(\mathrm{P}=0.0026)$ and 3 months after surgery $(\mathrm{P}<0.0001)$. However, no statistically significant difference was identified in any of the other regions between the two groups at the same time-windows. The significant variation distributions for subjects in the two groups are provided in Fig. 4.

There was no obvious statistically significant difference in the CT variation in each region between the high-myopia group and the super-high myopia group at 1 week. The CT variation between the pre-operative time-point and 1-month post-operatively was also similar in the two groups, but an increasing tendency for CT variation with the development of the degree of myopia was also observed at these times.

\section{Discussion}

ICL has been demonstrated to be an effective surgical treatment for the correction of refractive errors, offering good optical quality (14-17). With the expanding use of ICLs worldwide, concerns regarding the impact of the operation have grown. However, no data exist regarding the possible effects of ICL surgery on the choroid. To assess the potential influence of ICL surgery on the choroid, morphological changes in the status of the choroid were evaluated. The present study first reported that the choroid became thicker following ICL surgery in patients with high myopia. The CT in the foveal and nasal areas, as observed by SS-OCT, was significantly increased at $2 \mathrm{~h}$ after the surgery and reached 
Table I. Demographic and clinical information of the subjects.

\begin{tabular}{|c|c|c|c|}
\hline Variable & High-myopia group $(\mathrm{n}=42)$ & Super-high myopia group $(\mathrm{n}=56)$ & P-value \\
\hline Age (years) & $25.88 \pm 4.04$ & $26.45 \pm 5.02$ & 0.227 \\
\hline Sex (female/male) & $26 / 16$ & $36 / 20$ & 0.654 \\
\hline \multicolumn{4}{|l|}{ SE (D) } \\
\hline Pre-operative & $-7.91 \pm 1.37$ & $-14.91 \pm 2.17$ & 0.002 \\
\hline $1 \mathrm{w}$ post-operatively & $-0.53 \pm 0.29$ & $-0.73 \pm 0.13$ & 0.171 \\
\hline $1 \mathrm{~m}$ post-operatively & $-0.56 \pm 0.37$ & $-0.62 \pm 0.37$ & 0.774 \\
\hline $3 \mathrm{~m}$ post-operatively & $-0.31 \pm 0.32$ & $-0.41 \pm 0.32$ & 0.531 \\
\hline Axial length (mm) & $25.59 \pm 0.92$ & $27.74 \pm 1.12$ & 0.053 \\
\hline \multicolumn{4}{|l|}{ IOP (mmHg) } \\
\hline Pre-operatively & $13.34 \pm 0.56$ & $13.73 \pm 0.82$ & 0.914 \\
\hline $2 \mathrm{~h}$ post-operatively & $16.17 \pm 0.89$ & $16.83 \pm 0.77$ & 0.878 \\
\hline \multicolumn{4}{|l|}{ Anterior chamber } \\
\hline Washing time (sec) & 20 & 20 & $<0.001$ \\
\hline
\end{tabular}

a peak at 3 months. Comparing with preoperative, there was also a slightly increased thickness at 1 week and 1 month after surgery in the foveal and perifoveal choroid in the two groups. Only the changes in the fovea and nasal CT were statistically significant at $2 \mathrm{~h}$ and 3 months, suggesting that the minimum values measured around the central fovea were inadequate to evaluate the whole macula.

The effect of various refractive surgeries on $\mathrm{CT}$ has been reported in recent years $(18,19)$. Previous studies suggested that increases in CT were statistically significant at 1 month post-operatively $(20,21)$. This was inconsistent with the present results, which demonstrated a peak at 3 months; however, the trend of post-operative thickening was consistent. On the basis of the present results, the CTs exhibited a tendency of becoming thicker after correcting the refractive error. The proposed physiological mechanisms by which the choroid may dynamically increase or decrease in thickness include contraction and relaxation of nonvascular smooth muscle (22), fluid redistribution as a result of osmotic changes (23) and changes in choroidal blood flow (24); an association between CT and choroidal blood flow has been reported in animals (24) and humans $(25,26)$.

The present study suggested that the CT significantly increased at $2 \mathrm{~h}$ after the surgery, particularly in the fovea. This may be linked to inflammation and vascular permeability after surgery. It is known that surgical trauma induces the release of prostaglandins in the aqueous humor, which causes damage to the blood/aqueous barrier. This results in the accumulation of other inflammatory mediators, including endotoxins and immune complexes in the aqueous humor (27). These may diffuse into the vitreous cavity and reach the retina, leading to a rupture of the inner blood/retinal barrier, resulting in another cascade of inflammatory mediator secretions together with increased permeability of perifoveal capillaries $(27,28)$. Vascular permeability may have a role in changes in the CT. The proteins transported into the extracellular matrix and/or lymphatic system, followed by increased vascular permeability, may cause a large volume of liquid to move to the choroid, which may result in choroid thickening and restoration of vision in the form of deprivation myopia $(29,30)$. The outer blood/retinal barrier has also been indicated to be disrupted as a consequence of post-cataract surgery inflammation (31). Thus, the type of inflammation encountered in the posterior segment may also occur in the anterior segment. Xu et al (32) studied the effect of cataract surgery on the choroid and investigated the presence of factors that may affect the blood/retinal barrier, including interleukin-1 $\beta$, stromal cell-derived factor-1 and vascular endohelial growth factor. They indicated that surgical trauma induces an obvious inflammatory response, which accounts for the acute (30 min after surgery) inflammatory gene transcription observed in the retina (32). It may be hypothesized that similar changes occur in ICL implantation and cataract surgery. As these two surgeries are intraocular surgeries with lens implantation, they may cause disturbances to the internal eye environment and post-operative inflammation. Blood pressure changes caused by tension during the operation or the increase of IOP caused by anterior chamber washing and injection of viscoelastic material may also affect this. In the present study, the intraocular pressure measured $2 \mathrm{~h}$ after surgery increased by $\sim 3 \mathrm{mmHg}$ compared with that prior to surgery.

Furthermore, the subfoveal CT in the high-myopia group changed from $203.18 \pm 36.20 \mu \mathrm{m}$ pre-operatively to $205.51 \pm 36.04 \mu \mathrm{m}$ at 1 week post-operatively to $209.86 \pm 36.71 \mu \mathrm{m}$ at 1 month post-operatively and to $227.43 \pm 35.64 \mu \mathrm{m}$ at 3 months post-operatively. Consistently, the subfoveal CT in the super-high myopia group changed from $180.24 \pm 42.89 \mu \mathrm{m}$ pre-operatively to $181.88 \pm 40.14 \mu \mathrm{m}$ at 1 week post-operatively to $183.69 \pm 38.85 \mu \mathrm{m}$ at 1 month post-operatively and to $219.38 \pm 44.53 \mu \mathrm{m}$ at 3 months post-operatively. Similar results were obtained in the nasal area. CT increased slightly at 1 week and 1 month 

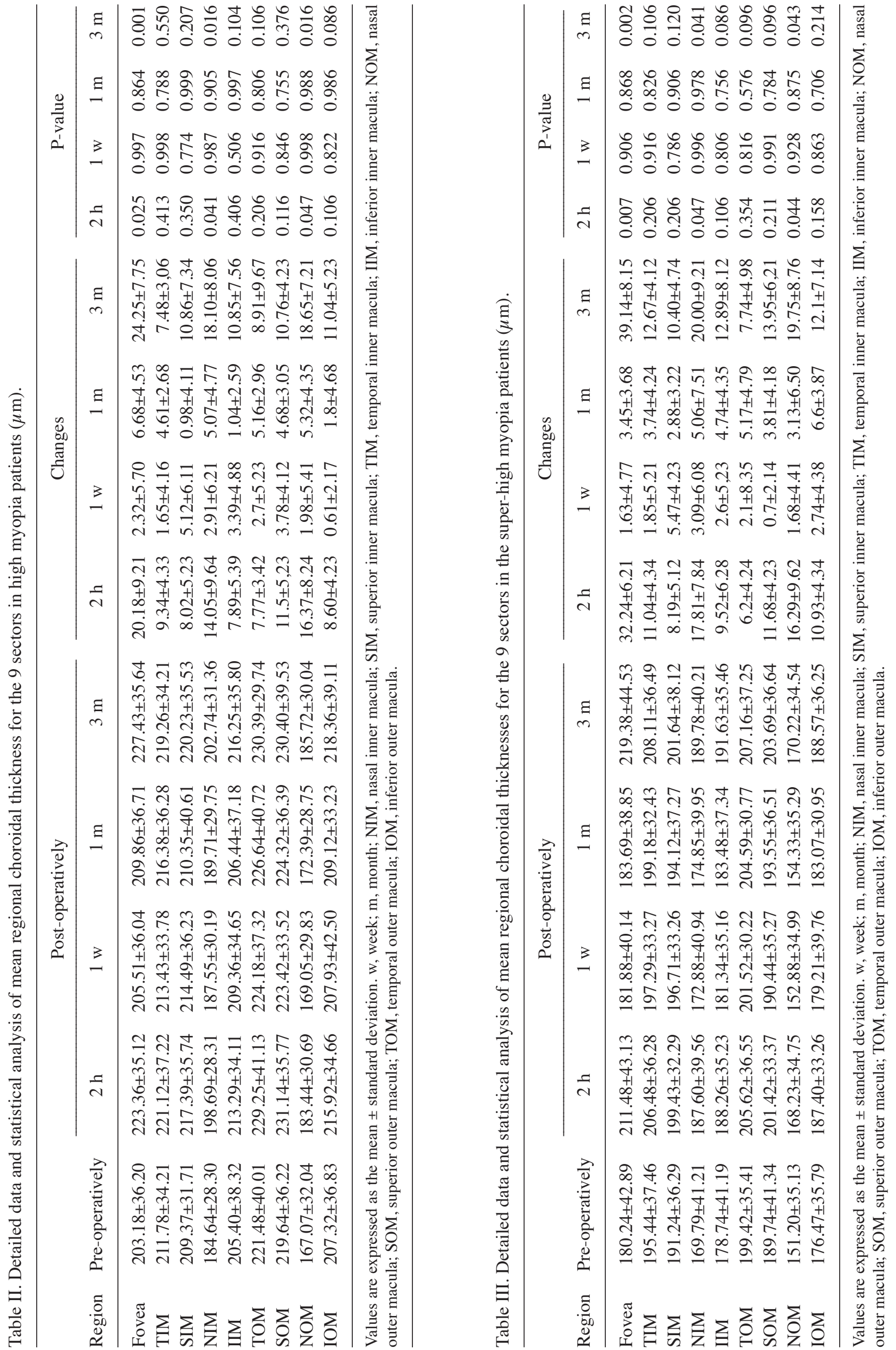

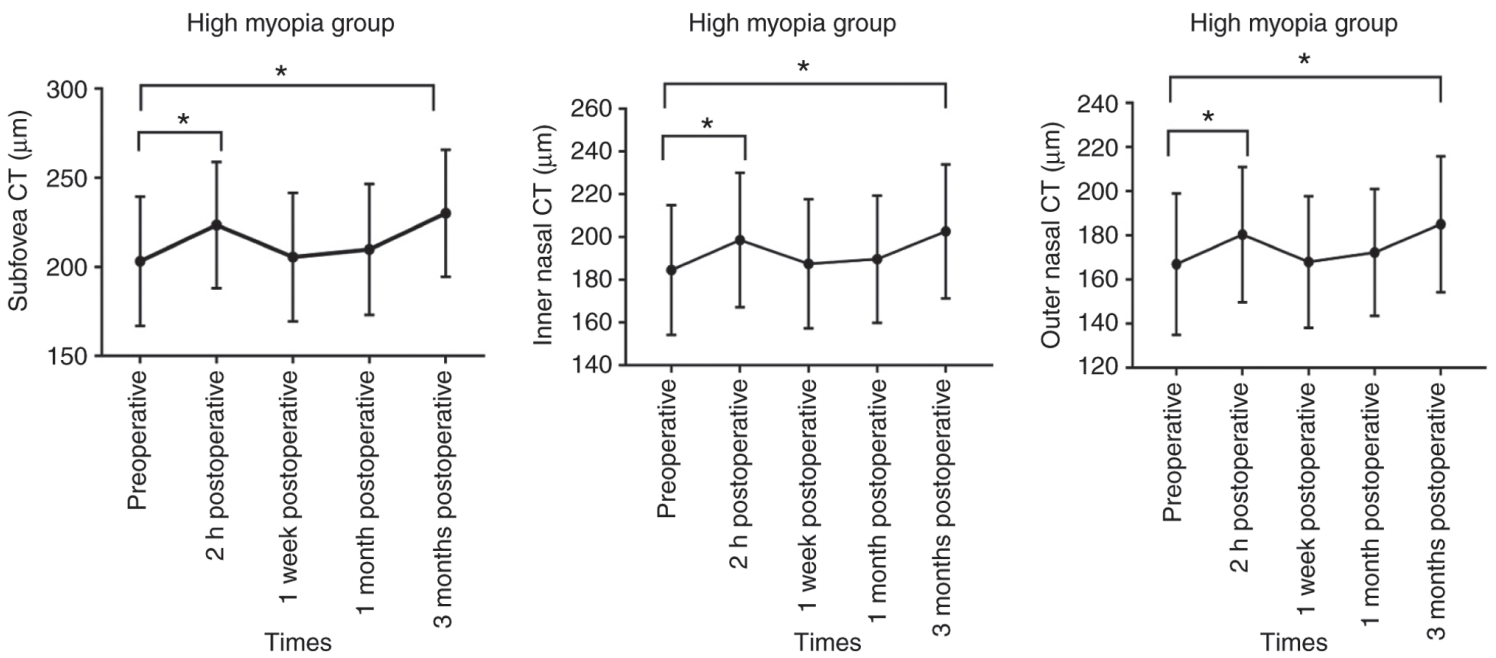

Figure 2. CT of the foveal and nasal side at different time-points in the high-myopia group. Values are expressed as mean \pm standard deviation. ${ }^{*} \mathrm{P}<0.05$. CT, choroidal thickness.
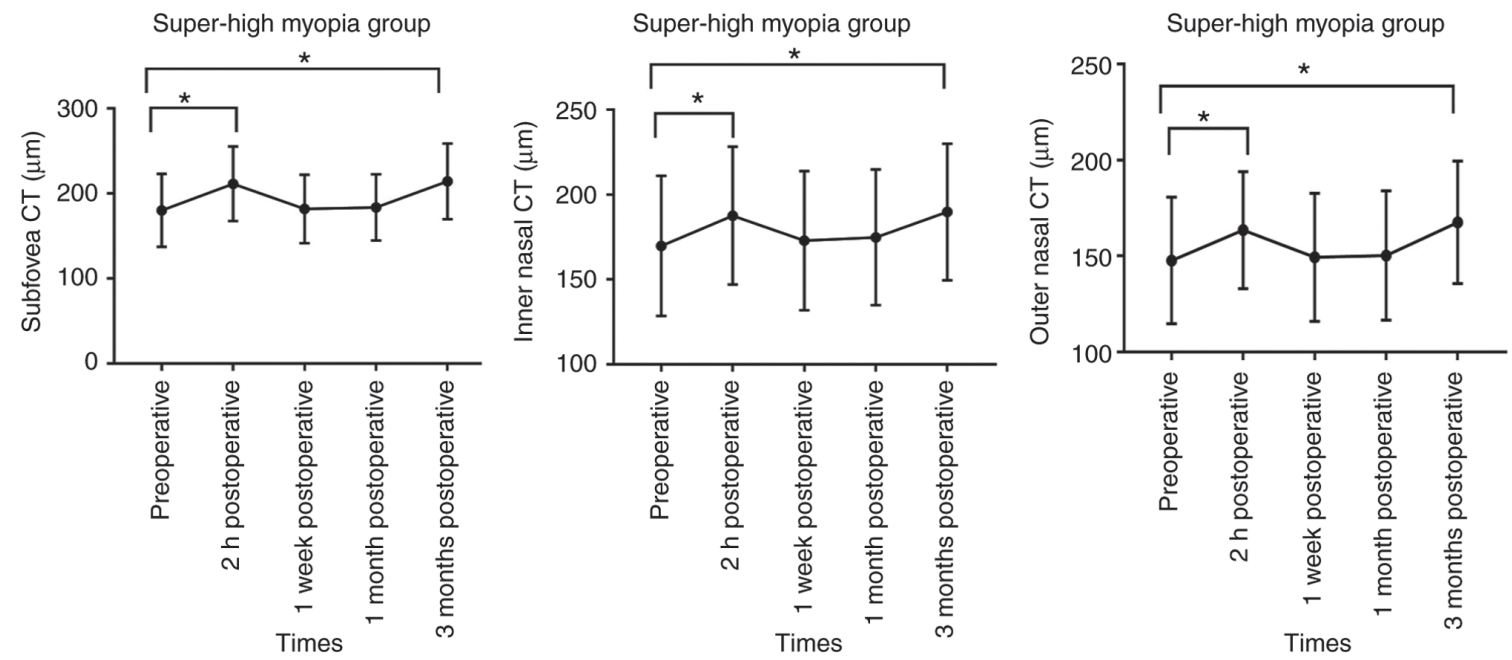

Figure 3. CT thickness of the fovea and nasal side at different time-points in the super-high myopia group. Values are expressed as mean \pm standard deviation. ${ }^{*} \mathrm{P}<0.05$. CT, choroidal thickness.
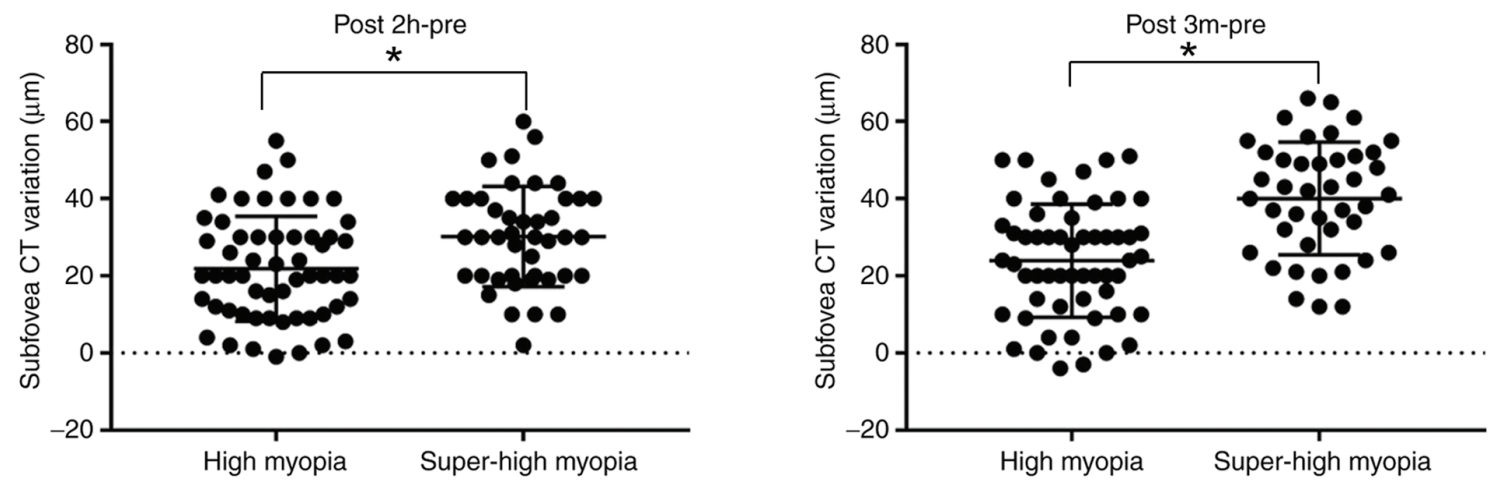

Figure 4. Changes in subfoveal $\mathrm{CT}$ in the two groups at $2 \mathrm{~h}$ and 3 months post-operatively vs. pre-operatively. Each data-point indicates the measurement value for one subject. Differences in $\mathrm{CT}$ variation between the two groups: $2 \mathrm{~h}$ after ICL surgery $(\mathrm{P}=0.0026)$ and 3 months after surgery $(\mathrm{P}<0.0001)$. $\mathrm{P}<0.05$. CT, choroidal thickness.

post-operatively but decreased when compared with that $2 \mathrm{~h}$ post-operatively. This suggested that there was a recovery period of $\mathrm{CT}$ after the operation. In the next month after surgery, the choroid gradually recovered, which is probably 
Table IV. Inter-group comparison of pre-operative and post-operative choroidal thickness variation for the 9 sectors.

\begin{tabular}{|c|c|c|c|c|}
\hline Subfield & $\begin{array}{c}\text { Pre-operatively to } \\
2 \mathrm{~h} \text { post-operatively }\end{array}$ & $\begin{array}{l}\text { Pre-operatively to } \\
1 \mathrm{w} \text { post-operatively }\end{array}$ & $\begin{array}{c}\text { Pre-operatively to } \\
1 \mathrm{~m} \text { post-operatively }\end{array}$ & $\begin{array}{l}\text { Pre-operatively to } \\
3 \text { m post-operatively }\end{array}$ \\
\hline \multicolumn{5}{|l|}{ Fovea } \\
\hline High myopia & $20.18 \pm 9.21$ & $2.32 \pm 5.70$ & $6.68 \pm 4.53$ & $24.25 \pm 7.75$ \\
\hline Super-high myopia & $32.24 \pm 6.21$ & $1.63 \pm 4.77$ & $3.45 \pm 3.68$ & $39.14 \pm 8.15$ \\
\hline P-value & 0.005 & 0.766 & 0.546 & 0.008 \\
\hline \multicolumn{5}{|l|}{ TIM } \\
\hline High myopia & $9.34 \pm 4.33$ & $1.65 \pm 4.16$ & $4.61 \pm 2.68$ & $7.48 \pm 3,06$ \\
\hline Super-high myopia & $11.04 \pm 4.34$ & $1.85 \pm 5.21$ & $3.74 \pm 4.24$ & $12.67 \pm 4.12$ \\
\hline P-value & 0.877 & 0.988 & 0.759 & 0.436 \\
\hline \multicolumn{5}{|l|}{ TOM } \\
\hline High myopia & $7.77 \pm 3.42$ & $2.7 \pm 5.23$ & $5.16 \pm 2.96$ & $8.91 \pm 9.67$ \\
\hline Super-high myopia & $6.20 \pm 4.24$ & $2.10 \pm 8.35$ & $5.17 \pm 4.79$ & $7.74 \pm 4.98$ \\
\hline P-value & 0.761 & 0.938 & 0.999 & 0.869 \\
\hline \multicolumn{5}{|l|}{ NIM } \\
\hline High myopia & $14.05 \pm 9.64$ & $2.91 \pm 6.21$ & $5.07 \pm 4.77$ & $18.10 \pm 8.06$ \\
\hline Super-high myopia & $17.81 \pm 7.84$ & $3.09 \pm 6.08$ & $5.06 \pm 7.51$ & $20.00 \pm 9.21$ \\
\hline P-value & 0.684 & 0.726 & 0.998 & 0.436 \\
\hline \multicolumn{5}{|l|}{ NOM } \\
\hline High myopia & $16.37 \pm 8.24$ & $1.98 \pm 5.41$ & $5.32 \pm 4.35$ & $18.65 \pm 7.21$ \\
\hline Super-high myopia & $16.29 \pm 9.62$ & $1.68 \pm 4.41$ & $3.13 \pm .6 .50$ & $19.75 \pm 8.76$ \\
\hline P-value & 0.782 & 0.865 & 0.548 & 0.782 \\
\hline \multicolumn{5}{|l|}{ SIM } \\
\hline High myopia & $8.02 \pm 5.23$ & $5.12 \pm 6.11$ & $0.98 \pm 4.11$ & $10.86 \pm 7.34$ \\
\hline Super-high myopia & $8.19 \pm 5.12$ & $5.47 \pm 4.23$ & $2.88 \pm 3.22$ & $10.40 \pm 4.74$ \\
\hline P-value & 0.934 & 0.896 & 0.519 & 0.967 \\
\hline \multicolumn{5}{|l|}{ SOM } \\
\hline High myopia & $11.5 \pm 5,23$ & $3.78 \pm 4.12$ & $4.68 \pm 3.05$ & $10.76 \pm 4.23$ \\
\hline Super-high myopia & $11.68 \pm 4.23$ & $0.70 \pm 2.14$ & $3.81 \pm 4.18$ & $13.95 \pm 6,21$ \\
\hline P-value & 0.946 & 0.467 & 0.774 & 0.793 \\
\hline \multicolumn{5}{|l|}{ IIM } \\
\hline High myopia & $7.89 \pm 5.39$ & $3.39 \pm 4.88$ & $1.04 \pm 2.59$ & $10.85 \pm 7.56$ \\
\hline Super-high myopia & $9.52 \pm 6.28$ & $2.6 \pm 5,23$ & $4.74 \pm 4.35$ & $12.89 \pm 8.12$ \\
\hline P-value & 0.767 & 0.856 & 0.593 & 0742 \\
\hline \multicolumn{5}{|l|}{ IOM } \\
\hline High myopia & $8.60 \pm 4.23$ & $0.61 \pm 2.17$ & $1.8 \pm 4.68$ & $11.04 \pm 5.23$ \\
\hline Super-high myopia & $10.93 \pm 4.34$ & $2.74 \pm 4.38$ & $6.6 \pm 3.87$ & $12.1 \pm 7.14$ \\
\hline P-value & 0.743 & 0.697 & 0.412 & 0.849 \\
\hline
\end{tabular}

Values are expressed as the mean \pm standard deviation. w, week; m, month; NIM, nasal inner macula; SIM, superior inner macula; TIM, temporal inner macula; IIM, inferior inner macula; NOM, nasal outer macula; SOM, superior outer macula; TOM, temporal outer macula; IOM, inferior outer macula.

associated with the decrease of intraocular inflammation and vascular permeability. At 3 months, a marked improvement in visual quality was noted.

The ciliary muscles and choroid are part of the accommodative apparatus of the eye. Ciliary muscles have been reported to extend into regions of the anterior choroid (33). As reported previously, the force involved in the contraction of the ciliary muscle may be transmitted to the choroid and the
CT may be affected by this mechanical force (34). Previous studies speculated that, in the absence of a clear retinal image, ocular accommodation was stimulated (35-37). It may be proposed that the refractive error was corrected and retinal imaging quality was at its clearest and most stable at 3 months after surgery; this may reduce the accommodative demand. Ultimately, the choroid becomes thicker following the decrease in the tension on the ciliary muscle and the stretching of the 
eyeball attached to the choroid. This was different from the observed thickening at 1 month post-operatively. It may be speculated that the follow-up time of previous studies was not as long as that in the present study, or that the retinal quality of patients with high myopia was poorer than that of normal eyes, and therefore, the patients took longer to recover to achieve better visual quality. Furthermore, in the choroidal map, significant changes in CT were only present in nasal regions, except for the thickening of the foveal CT. The mechanism remains elusive, but may be linked to accommodation, which is mainly regulated by the ciliary muscle and ligaments of the lens. The ciliary muscle moves forward and inward, allowing the lens equator to move away from the sclera by stretching of the choroid (38). A previous study indicated that, during the accommodation process, the ciliary muscle mainly stretched horizontally (19). Furthermore, the basal CT of the nasal side was thinner than that of other areas, and a thinner area was associated with a greater impact, just as indicated by the choroidal changes of the super-myopia group.

In the present study, it was observed that there were significant differences in subfoveal CT variations between the two groups. The subfoveal CT exhibited a greater increase in the super-high-myopia group compared with that in the high-myopia group at $2 \mathrm{~h}$ and at 3 months post-operatively. It may be speculated that, with the increase in the degree of myopia, the improvement of imaging after retinal surgery may be more obvious than prior to retinal surgery, and that patients with super-high myopia may be more sensitive to choroidal vascular osmotic pressure changes. The refractive error was corrected and ocular accommodation was reduced, followed by the choroid becoming thicker. Super-high myopia requires greater pre-operative refractive power, which reduces after surgery. This suggested that the degree of CT appeared to be moderately correlated with the degree of refractive power for the subjects. Previous studies demonstrated that structural changes in the choroid may be explained by visual information being transferred through neurotransmitters from the retina to the choroid following visual recovery after surgery $(39,40)$. At present, the mechanism remains elusive.

The present study has several limitations. First, the study was limited by the relatively short follow-up period, so it is not known how long the effects may last once the refractive error was corrected. Intraoperative blood pressure and IOP were not recorded. Furthermore, no detailed comparison of the potential effects of diopter differences at different time-points after surgery was made. Although further studies are required to confirm the present observations, the present study reported on CT changes following ICL surgery and compared the variations between patients with different degrees of myopia. The present study also had the largest sample size among studies published thus far.

In conclusion, the present study observed that $\mathrm{CT}$ significantly increased at $2 \mathrm{~h}$ after surgery and reached a peak at 3 months after surgery, particularly in the subfoveal and nasal areas. The CT was significantly increased in the super-high myopia group. CT precedes the occurrence of numerous retinal abnormalities. Consequently, it may be anticipated that further research will focus on the thickening of the cornea after ICL surgery. The present results may enable us to better evaluate the post-operative safety of patients with high myopia and super-high myopia.

\section{Acknowledgements}

We thank Yan Liu (MD, PhD) for providing insight and expertise in the early stages of the research. We thank our colleague Zhenzhen Zhang (MD, PhD) for assistance and comments that greatly improved the manuscript. We would also like to express our gratitude to Xuefei Song (MD, PhD) for his experience in statistical analysis.

\section{Funding}

This study was supported by the National Natural Science Foundation of China (grant nos. 81570884 and 81770961), the Science and Technology Commission of Shanghai (grant no. 17DZ2260100) and Seed Founding of Shanghai Ninth People's Hospital, Shanghai JiaoTong University School of Medicine (grant no. JYZZ001).

\section{Availability of data and materials}

The datasets used and/or analyzed during the current study are available from the corresponding author on reasonable request.

\section{Authors' contributions}

$\mathrm{JZ}$ and RJ analyzed and interpreted the patient data regarding the high myopia patients. JY performed the ocular examination and FH was responsible for study design and was a major contributor in writing the manuscript. All authors read and approved the final manuscript.

\section{Ethics approval and consent to participate}

Written informed consent regarding the use of data for scientific research was provided by all subjects. The study was approved by the University Investigational Review Board (Shanghai, China).

\section{Patient consent for publication}

Not applicable.

\section{Competing interests}

The authors declare that they have no competing interests.

\section{References}

1. Williams KM, Hysi PG, Nag A, Yonova-Doing E, Venturini C and Hammond CJ: Age of myopia onset in a British populationbased twin cohort. Ophthalmic Physiol Opt 33: 339-345, 2013.

2. Igarashi A, Shimizu K and Kamiya K: Eight-year follow-up of posterior chamber phakic intraocular lens implantation for moderate to high myopia. Am J Ophthalmol 157: 532-539, 2014.

3. Sari ES, Pinero DP, Kubaloglu A, Evcili PS, Koytak A, Kutluturk I and Ozerturk Y: Toric implantable collamer lens for moderate to high myopic astigmatism: 3-year follow-up. Graefes Arch Clin Exp Ophthalmol 251: 1413-1422, 2013. 
4. Gyldenkerne A, Ivarsen A and Hjortdal JO: Comparison of corneal shape changes and aberrations induced By FS-LASIK and SMILE for myopia. J Refract Surg 31: 223-229, 2015

5. Gao S, Li S, Liu L, Wang Y, Ding H, Li L and Zhong X: Early changes in ocular surface and tear inflammatory mediators after small incision lenticule extraction and femtosecond laser-assistedlaser in situ keratomileusis. PLoS One 9: e107370, 2014

6. Flores-Moreno I, Lugo F, Duker JS and Ruiz-Moreno JM: The relationship between axial length and choroidal thickness in eyes with high myopia. Am J Ophthalmol 155: 314-319. e1, 2013.

7. Ho M, Liu DT, Chan VC and Lam DS: Choroidal thickness measurement in myopic eyes by enhanced depth optical coherence tomography. Ophthalmology 120: 1909-1914, 2013.

8. Gupta P, Cheung CY, Saw SM, Bhargava M, Tan CS, Tan M, Yang A, Tey F, Nah G, Zhao P, et al: Peripapillary choroida thickness in young Asians with high myopia. Invest Ophthalmo Vis Sci 56: 1475-1481, 2015.

9. Grossniklaus HE and Green WR: Choroidal neovascularization. Am J Ophthalmol 137: 496-503, 2004.

10. Fitzgerald ME, Wildsoet CF and Reiner A: Temporal relationship of choroidal blood flow and thickness changes during recovery from form deprivation myopia in chicks. Exp Eye Res 74: 561-570, 2002.

11. Rada JA and Palmer L: Choroidal regulation of scleral glycosaminoglycan synthesis during recovery from induced myopia. Invest Ophthalmol Vis Sci 48: 2957-2966, 2007.

12. Swarbrick HA, Alharbi A, Watt K, Lum E and Kang P: Myopia control during orthokeratology lens wear in children using a novel study design. Ophthalmology 122: 620-630, 2015.

13. Tang Y, Wang X, Wang J, Jin L, Huang W, Luo Y and Lu Y: Risk factors of age-related cataract in a Chinese adult population: The Taizhou Eye Study. Clin Exp Ophthalmol 46: 371-379, 2018.

14. Kamiya K, Shimizu K, Igarashi A, Hikita F and Komatsu M: Four-year follow-up of implantable collamer lens implantation for moderate to high myopia. Arch Ophthalmol 127: 845-850, 2009.

15. Ju Y, Gao XW and Ren B: Posterior chamber phakic intraocular lens implantation for high myopia. Int J phthalmol 6: 831-835, 2013.

16. Fernández-Vigo JI, Macarro-Merino A, Fernández-Vigo C Fernández-Vigo JÁ, Martínez-de-la-Casa JM, Fernández-Pérez C and García-Feijóo J: Effects of implantable Collamer lensV4c placement on iridocorneal angle measurements by Fourier-domain optical coherence tomography. Am J Ophthalmol 162: 43-52.e1, 2016.

17. Ju Y, Gao XW and Ren B: Posterior chamber phakic intraocular lens implantation for high myopia. Int J Ophthalmol 6: 831-835, 2013.

18. Pierru A, Carles M, Gastaud P and Baillif S: Measurement of subfoveal choroidal thickness after cataract surgery in enhanced depth imaging optical coherence tomography. Invest Ophthalmol Vis Sci 55: 4967-4974, 2014.

19. Li M, Cheng H, Yuan Y, Wang J, Chen Q, Me R and Ke B: Change in choroidal thickness and the relationship with accommodation following myopic excimer laser surgery. Eye (Lond) 30: 972-978, 2016.

20. Falcão MS, Gonçalves NM, Freitas-Costa P, Beato JB, Rocha-Sousa A, Carneiro A, Brandão EM and Falcão-Reis FM: Choroidal and macular thickness. Changes induced by cataract surgery. Clin Ophthalmol 8: 55-60, 2014

21. Biro Z and Balla Z: OCT measurements on the foveal and perifoveal retinal thickness on diabetic patients after phacoemulsification and IOL implantation. Eye (Lond) 24: 639-647, 2010.

22. Nickla DL and Wallman J: The multifunctional choroid. ProgRetin Eye Res 29: 144-168, 2010.
23. Wallman J, Wildsoet C, Xu A, Gottlieb MD, Nickla DL, Marran L, Krebs W and Christensen AM: Moving the retina: Choroidal modulation of refractive state. Vision Res 35: 37-50, 1995.

24. Fitzgerald ME, Wildsoet $\mathrm{CF}$ and Reiner A: Nasal relationship of choroidal blood flow and thickness changes during recovery from form deprivation myopia in chicks. Exp Eye Res 74: 561-570, 2002.

25. Novais EA, Badaro E, Allemann N, Morales MS, Rodrigues EB, de Souza Lima R, Regatieri CV and Belfort R Jr: Correlation between choroidal thickness and ciliary artery blood flow velocity in normal subjects. Ophthalmic Surg Lasers Imaging Retina 46: 920-924, 2015

26. Kim DY, Silverman RH, Chan RV, Khanifar AA, Rondeau M, Lloyd H, Schlegel P and Coleman DJ: Measurement of choroidal perfusion and thickness following systemic silde-nafil (Viagrat (囚)). Acta Ophthalmol 91: 183-188, 2013.

27. Miyake K and Ibaraki N: Prostaglandins and cystoid macular edema. Survey Ophthalmol 47: S203-S218, 2002.

28. Flach AJ: The incidence, pathogenesis and treatment of cystoid macular edema following cataract surgery. Trans Am Ophthalmol Soc 96: 557-634, 1998.

29. Pendrak K, Papastergiou GI, Lin T, Laties AM and Stone RA Choroidal vascular permeability in visually regulated eye growth. Exp Eye Res 70: 629-637, 2000.

30. Junghans BM, Crewther SG, Liang H and Crewther DP: A role for choroidal lymphatics during recovery from form deprivation myopia? Optom Vis Sci 76: 796-803, 1999.

31. Tso MO and Shih CY: Experimental macular edema after lens extraction. Invest Ophthalmol Vis Sci 16: 381-392, 1977.

32. Xu H, Chen M, Forrester JV and Lois N: Cataract surgery induces retinal pro-inflammatory gene expression and protein secretion. Invest Ophthalmol Vis Sci 52: 249-255, 2011.

33. Jeon S, Lee WK, Lee K and Moon NJ: Diminished ciliary muscle movement on accommodation in myopia. Exp Eye Res 105: 9-14, 2012.

34. Woodman EC, Read SA and Collins MJ: Axial length and choroidal thickness changes accompanying prolonged accommodation in myopes and emmetropes. Vis Res 72: 34-41, 2012.

35. Pandian A, Sankaridurg PR, Naduvilath T, O'Leary D, Sweeney DF, Rose K and Mitchell P: Accommodative facility in eyes with and without myopia. Invest Ophthalmol Vis Sci 47: 4725-4731, 2006

36. Sreenivasan V, Aslakson E, Kornaus A and Thibos LN: Retinal image quality during accommodation in adult myopic eyes. Optom vis sci 90: 1292-1303, 2013.

37. Lee AG, Kohnen T, Ebner R, Bennett JL, Miller NR, Carlow TJ and Koch DD: Optic neuropathy associated with laser in situ keratomileusis. J Cataract Refract Surg 26: 1581-1584, 2000.

38. Croft MA, Glasser A, Heatley G, McDonald J, Ebbert T, Dahl DB, Nadkarni NV and Kaufman PL: Accommodative ciliary body and lens function in rhesus monkeys, I: normal lens, zonule and ciliary process configuration in the iridectomized eye. Invest Ophthalmol Vis Sci 47: 1076-1086, 2006.

39. Seko Y, Tanaka Y and Tokoro T: Apomorphine inhibits the growth-stimulating effect of retinal pigment epithelium on scleral cells in vitro. Cell Biochem Funct 15: 191-196, 1997.

40. Wallman J, Gottlieb MD, Rajaram V and Fugate-Wentzek LA Local retinal regions control local eye growth and myopia. Science 237: 73-77, 1987.

This work is licensed under a Creative Commons Attribution-NonCommercial-NoDerivatives 4.0 International (CC BY-NC-ND 4.0) License. 